\title{
Region Segmentation Using Information Divergence Measures
}

\author{
Lyndon S. Hibbard \\ Computerized Medical Systems, Inc., St. Louis, MO 63132, USA, \\ lyn@cms-stl.com
}

\begin{abstract}
Image segmentations based on maximum likelihood (ML) or maximum a posteriori (MAP) analyses of object textures usually assume parametric models (e.g., Gaussian) for distributions of these features. For real images, parameter accuracy and model stationarity may be elusive, so that model-free inference methods ought to have an advantage over those that are model-dependent. Functions of the relative entropy (RE) from information theory can produce minimum error, model-free inferences, and can detect the boundary of an image object by maximizing the $\mathrm{RE}$ between the pixel distributions inside and outside a flexible curve contour. A generalization of the RE-the Jensen-Rényi divergence (JRD)computes optimal $n$-way decisions and can contour multiple objects in an image simultaneously. Seed regions expand naturally and multiple contours tend not to overlap. We apply these functions to contour patient anatomy in X-ray computed tomography (CT) for radiotherapy treatment planning.
\end{abstract}

\section{Introduction}

Segmentation of discrete objects in digital medical images continues to be an important problem across modalities and applications. We are concerned with contouring patient anatomy in CT images to create individual radiotherapy cancer treatments. Anatomy contouring is costly because the anatomy is complex and often appears at low contrast or blurred by organ motion. Expert interpretation and manual rendering is often required. Automated segmentation would reduce the cost and subjectivity of the contour map, leaving to the operator the tasks of reviewing the contours and editing them as appropriate.

This article describes novel criteria for region segmentation, all based on the relative entropy ( $\mathrm{RE}$ ) of information theory. The RE is a measure of the differences between probablity distributions. By maximizing the RE between pixels inside and outside a flexible closed curve contour, one can approximate the boundary between those regions. The Jensen-Rényi divergence (JRD) is a generalization of the RE that produces optimal, $n$-way decisions that enable contouring of several regions in an image simultaneously.

Information theory has been mostly applied to digital data coding and compression, but image analysis applications have appeared recently in greater numbers (see the review by O'Sullivan etal.[17]). The August 2000 issue of the IEEE 
Transactions on Information Theory was entirely devoted to image analysis. Mutual information for image registration [1 15 24 25 26] is surely one of the most important developments in recent years. Information divergence measures (RE and variants) have been recently applied to tomographic image reconstruction with artifact modeling 222 23], target-background contrast measurement [6], MRI segmentation [8], and image edge detection [9].

In this paper, we review optimal classification and the information theoretic equivalents to the likelihood ratio test, and demonstrate region segmentations based on them.

\section{Optimal Classification}

\subsection{Minimum-Error Decision Rules}

Consider the discrete pixel random variable $X$ that takes values from a finite integer set $X=x \in\left\{x_{1}, \ldots, x_{N}\right\}$, with probability distribution $p\left(x_{i}\right)=\operatorname{Pr}\{X=$ $\left.x_{i}\right\}$ where $0 \leq p\left(x_{i}\right) \leq 1$ for all $i$ and $\sum_{i=1}^{N} p\left(x_{i}\right)=1$. For a picture with $R$ regions $r_{1}, \ldots, r_{R}$, we are interested in the conditional probabilities $p\left(x \mid r_{j}\right)$ where $\sum_{i=1}^{N} p\left(x_{i} \mid r_{j}\right)=p\left(r_{j}\right)$ and $\sum_{j=1}^{R} \sum_{i=1}^{N} p\left(x_{i} \mid r_{j}\right)=1$. An observed $x$ is assigned to region $r^{*}$ according to $r^{*}=\arg \max _{j}\left(p\left(x \mid r_{j}\right)\right)$. If we have prior knowledge of the $p\left(r_{j}\right)$, Bayes theorem directs that $x$ be assigned to region $r^{*}=$ $\arg \max _{j}\left(p\left(x \mid r_{j}\right) p\left(r_{j}\right)\right)$ where the product $p\left(x \mid r_{j}\right) p\left(r_{j}\right)$ is proportional to the true a posteriori probability $p\left(r_{j} \mid x\right)$, or the probability that $r_{j}$ is the true region given the observation of $x$. Either rule-maximum likelihood (ML) or maximum a posteriori (MAP), respectively - guarantees that the resulting decisions will, on averge, produce minimum error $3 \sqrt{455}$. Since the distributions $p(\cdot)$ can be difficult to evaluate, ML and MAP inferences are often made by evaluating discriminant functions operating on parametric models of the data distributions.

\subsection{Relative Entropy and Optimal Classification}

Kullback and Leibler 1213 made the interesting observation that minimum error classification can be obtained from an examination of the differences between probability distributions using the RE (also known as the Kullback-Leibler divergence, the I-divergence, the cross entropy, etc.). The RE for two regionconditioned distributions $p\left(x \mid r_{1}\right), p\left(x \mid r_{0}\right)$ is $12 \mid 132$

$$
D\left(p\left(x \mid r_{1}\right) \| p\left(x \mid r_{0}\right)\right)=\sum_{i=1}^{N} p\left(x \mid r_{1}\right) \log _{2} \frac{p\left(x \mid r_{1}\right)}{p\left(x \mid r_{0}\right)}
$$

where $0 \log \left(0 / p_{0}\right)=0$. Terms in which $p_{0}=0$ are undefined and must be neglected to provide absolute continuity of $p_{1}$ with $p_{0}$ [13]. The RE is convex and $D\left(p_{1} \| p_{0}\right) \geq 0$ with $D\left(p_{1} \| p_{0}\right)=0$ when $p_{1}=p_{0}$ everywhere. The RE is a directed divergence since in general $D\left(p_{1} \| p_{0}\right) \neq D\left(p_{0} \| p_{1}\right)$. 


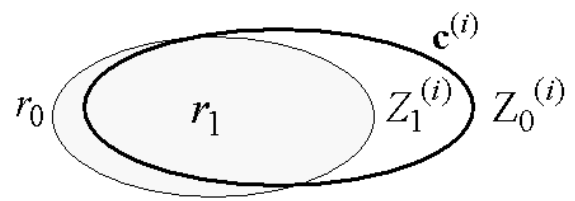

Fig. 1. Definition of the contour problem and nomenclature. $r_{1}$ is the object to be segmented from the background $r_{0}$. The boundary of $r_{1}$ is approximated by a flexible closed parametric curve $\mathbf{c}=\left[c_{1}, c_{2}, \ldots\right]^{T}$ fit to the $r_{1}$ boundary by maximizing an objective function over the coefficients $c_{i}$. $\mathbf{c}^{(i)}$ represents the $i$-th iterate during maximization with inside and outside zones $Z_{0}^{(i)}, Z_{1}^{(i)}$, respectively.

Consider now a sequence of samples $X_{1}, \ldots, X_{K}$ drawn independently and identically from either one of the two conditional distributions $p\left(X \mid r_{1}\right), p\left(X \mid r_{0}\right)$. The ML test for two outcomes (likelihood ratio test, LRT), is:

$$
\text { If } p\left(X_{1}, \ldots, X_{K} \mid r_{1}\right) / p\left(X_{1}, \ldots, X_{K} \mid r_{0}\right)>T \text {, decide } r_{1} \text {, else } r_{0},
$$

where threshold $T$ can be determined from training data. There exists a RE test equivalent to the LRT ([2], Chapter 12) in which the divergences between the distribution of the $X_{i}, p_{X}(x)$, and the region conditional distributions also produces an average minimum error decision:

$$
\text { If } D\left(p_{X}(x) \| p\left(x \mid r_{0}\right)\right)-D\left(p_{X}(x) \| p\left(x \mid r_{1}\right)\right)>\frac{1}{K} \log _{2} T \text {, decide } r_{1} \text {, else } r_{0} \text {. }
$$

This is a minimum-error classifier which assigns a sample distribution to one of two known distributions with no reference to a model for the distributions.

\section{Divergences and Optimal Contours}

\subsection{Relative Entropy and the Best Contour}

We turn now from classification of samples to finding the optimal partition separating samples from the two regions. In Figure 1, region $r_{1}$ is to be segmented from the background $r_{0}$ by a flexible curve $\mathbf{c}$ to be fit to the $r_{1}$ boundary by maximizing a divergence function of the curve parameters. Any c other than that which perfectly coincides with $r_{1}$ will have zones $Z_{i}$ overlapping with multiple regions $r_{j}$. The dependence of the random variable $X$ on the regions and zones is captured by the joint probability distribution $p(X, Z, r)$ which can be written as $p(X \mid Z, r) p(Z \mid r) p(r)$, the product of the conditional probability of observing $X$ given $Z$ and $r$, the conditional probability of being at a point in zone $Z$ given region $r$, and the (prior) probability $p(r)$ that a point is in region $r$. If the match of $\mathbf{c}$ to the $r_{1}$ boundary improves, the zones will approach the regions in size, shape, and pixel distribution, with $p\left(Z_{j} \mid r_{j}\right)>>p\left(Z_{j} \mid r_{i}\right)$ as $Z_{j} \rightarrow r_{j}, i \neq$ $j$. Since the $p\left(r_{i}\right)$ are constant, changes in $p(X, Z, r)$ will be a function only 
of the factors dependent on $Z, p\left(X \mid Z_{j}\right)$, and $p\left(Z_{j} \mid r_{j}\right)$. Therefore, a 2-zone, 2region LRT would involve ratios of the sort $\frac{p\left(X \mid Z_{1}\right) p\left(Z_{1} \mid r_{1}\right)}{p\left(X \mid Z_{0}\right) p\left(Z_{0} \mid r_{0}\right)}$. It can be shown by a derivation too lengthy for this setting that the LRT-equivalent objective function which maximizes the match of $\mathbf{c}$ to $r_{1}$ is exactly what one should expect,

$$
\mathbf{c}^{*}=\arg \max _{\mathbf{c}} D\left(p\left(X \mid Z_{1}\right)|| p\left(X \mid Z_{0}\right)\right) .
$$

\subsection{Shannon and Rényi Entropies}

The Shannon entropy $H_{S}(p)$ for the distribution $p(X)$ of the discrete random variable $X$ is 21

$$
H_{S}(p)=-\sum_{i=1}^{N} p\left(x_{i}\right) \log _{2} p\left(x_{i}\right)
$$

where $H_{S}(p) \geq 0$ and $H_{S}(p)=0$ exactly if all the probability is associated with one outcome $p\left(x_{i}\right)=1$, with all the other outcomes' probabilities $p\left(x_{j}\right)=0, j \neq$ $i$. $H_{S}(p)$ is a maximum when $p\left(x_{i}\right)=1 / N$ for all $i$.

Rényi introduced a generalization of the Shannon entropy [20]. The Rényi entropy of degree $\alpha$, is defined as

$$
H_{R \alpha}(p)=\frac{1}{1-\alpha} \log _{2} \sum_{i=1}^{N} p\left(x_{i}\right)^{\alpha} .
$$

The Rényi entropy is concave for $\alpha<1$ and tends to the Shannon entropy as $\alpha \rightarrow 1\left[20\right.$. The Shannon and Rényi entropies are related as [19] $H_{R \alpha} \geq H_{S} \geq$ $H_{R \beta}$ where $0<\alpha<1$ and $\beta>1$.

\subsection{Jensen-Shannon and Jensen-Rényi Divergences}

Two generalizations of the relative entropy exist. Lin 14 introduced the JensenShannon divergence (JSD) $J S_{\pi}$, defined here for the case of two regions $r_{0}, r_{1}$,

$$
J S_{\pi}(p)=H_{S}\left(\pi_{0} p\left(x \mid r_{0}\right)+\pi_{1} p\left(x \mid r_{1}\right)\right)-\pi_{0} H_{S}\left(p\left(x \mid r_{0}\right)\right)-\pi_{1} H_{S}\left(p\left(x \mid r_{1}\right)\right)
$$

where $H_{S}(p)$ is the Shannon entropy. $J S_{\pi}(p)=0$ when $p\left(x \mid r_{0}\right)=p\left(x \mid r_{1}\right)$ for all $x$, and increases as differences between the two distributions grow larger. The weights $\pi_{0}, \pi_{1}$ are defined such that $0 \leq \pi_{i} \leq 1$ for all $i$ and $\sum_{i} \pi_{i}=1$. The $J S_{\pi}$ is symmetric with respect to interchange of subscripts. The $J S_{\pi}$ can also be defined for $R$ regions [14]

$$
J S_{\pi}\left(p_{1}, \ldots, p_{R}\right)=H_{S}\left[\sum_{i=1}^{R} \pi_{i} p_{i}\right]-\sum_{i=1}^{R} \pi_{i} H_{S}\left(p_{i}\right)
$$

where the $p_{1}, \ldots, p_{R}$ are the $p\left(x \mid r_{1}\right), \ldots, p\left(x \mid r_{R}\right)$ and the $\pi_{i}$ are as noted above. $J S_{\pi}=0$ when $p\left(x \mid r_{1}\right)=p\left(x \mid r_{2}\right)=\cdots=p\left(x \mid r_{R}\right)$, and is maximum when the distributions are all maximally different one from another. 

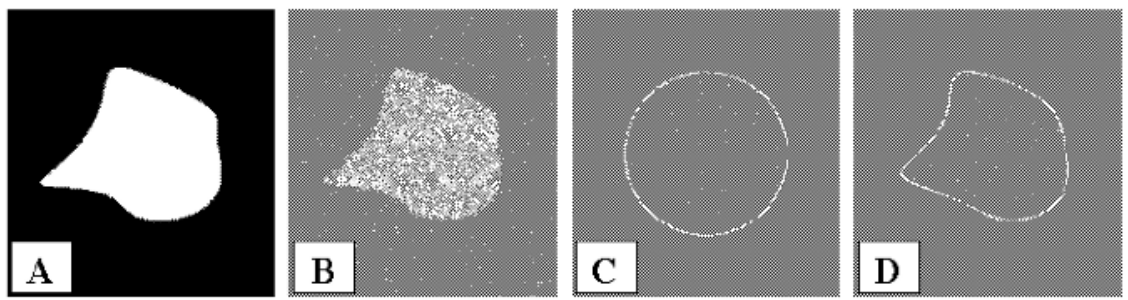

Fig. 2. Random shape grayscale figures for automated segmentation testing.

The Jensen-Rényi divergence (JRD) was introduced by He etal. [10] and is defined here for $R$ region distributions as

$$
J R_{\alpha}^{\pi}\left(p_{1}, \ldots, p_{R}\right)=H_{R \alpha}\left[\sum_{i=1}^{R} \pi_{i} p\left(x \mid r_{i}\right)\right]-\sum_{i=1}^{R} \pi_{i} H_{R \alpha}\left(p\left(x \mid r_{i}\right)\right)
$$

where the $p_{1}, \ldots, p_{R}$ are the $p\left(x \mid r_{1}\right), \ldots, p\left(x \mid r_{R}\right)$ and the $\pi_{i}$ are as noted above. $J R_{\alpha}^{\pi} \geq 0$, equality for $p\left(x \mid r_{1}\right)=p\left(x \mid r_{2}\right)=\cdots=p\left(x \mid r_{R}\right)$. The JRD is symmetric with respect to interchange of subscripts, and is convex in the $p_{1}, \ldots, p_{R}$ for $\alpha \in(0,1)\left[10 . J R_{\alpha}^{\pi} \rightarrow J S_{\pi}\right.$ as $\alpha \rightarrow 1$, so the JSD is a specialization of the JRD.

The JRD can be defined for multiple independent random variables $X_{1}, . ., X_{D}$ that are independent in the sense that $p\left(X_{1}, \ldots, X_{D} \mid r\right)=\prod_{d=1}^{D} p\left(X_{d} \mid r\right)$. The multivariate JRD for $R$ distributions is

$$
J R_{\alpha}^{\pi}\left(p_{1}, \ldots, p_{R}\right)=H_{R \alpha}\left[\sum_{i=1}^{R} \pi_{i} \sum_{d=1}^{D} p\left(X_{d} \mid r_{i}\right)\right]-\sum_{i=1}^{R} \pi_{i} \sum_{d=1}^{D} H_{R \alpha}\left(p\left(X_{d} \mid r_{i}\right)\right)
$$

\section{Results}

\subsection{Divergence Contour Objective Functions}

The contour best approximating a region boundary, $\mathbf{c}^{*}$, is that which maximizes the JRD over the zones inside $\left(Z_{1}\right)$ and outside $\left(Z_{0}\right)$ the contour

$$
\begin{aligned}
\mathbf{c}^{*}=\arg \max _{\mathbf{c}}\left[J R_{\alpha}^{\pi}\left(p_{0}, p_{1}\right)\right] \\
=\arg \max _{\mathbf{c}}\left[H_{R \alpha}\left(\pi_{0} p\left(x \mid Z_{0}\right)+\pi_{1} p\left(x \mid Z_{1}\right)\right)-\right. \\
\left.\quad \pi_{0} H_{R \alpha}\left(p\left(x \mid Z_{0}\right)\right)-\pi_{1} H_{R \alpha}\left(p\left(x \mid Z_{1}\right)\right)\right] .
\end{aligned}
$$

For $R$ regions, or $R-1$ objects plus the background (0), the set of best contours $\left\{\mathbf{c}_{1}^{*}, \mathbf{c}_{2}^{*}, \ldots, \mathbf{c}_{R-1}^{*}\right\}$ are those whose collective coefficients maximize the JRD

$$
\begin{aligned}
\mathbf{c}_{1}^{*}, \mathbf{c}_{2}^{*}, \ldots, \mathbf{c}_{R-1}^{*}= & \arg \max _{\mathbf{c}_{1}, \mathbf{c}_{2}, \ldots, \mathbf{c}_{R-1}} \\
& {\left[H_{R \alpha}\left(\sum_{i=0}^{R-1} \pi_{i} p\left(x \mid Z_{i}\right)\right)-\sum_{i=0}^{R-1} \pi_{i} H_{R \alpha}\left(p\left(x \mid Z_{i}\right)\right)\right] . }
\end{aligned}
$$




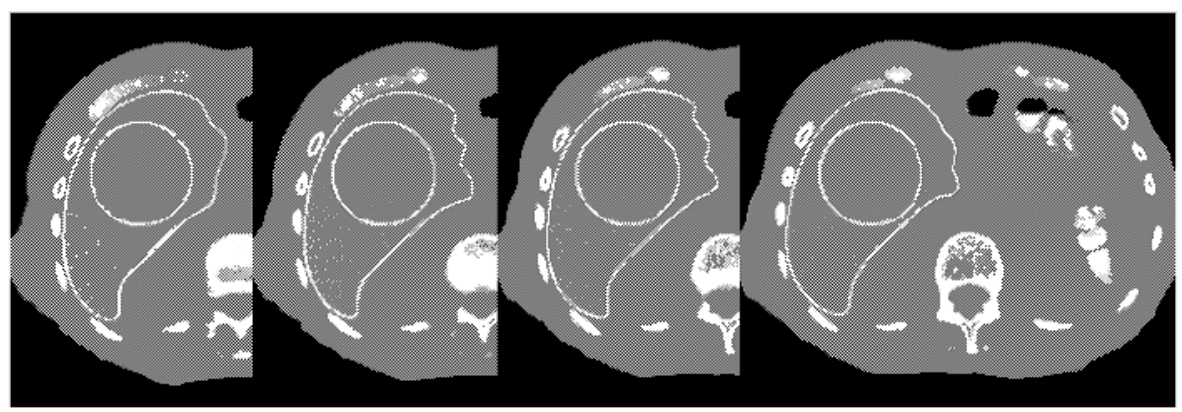

Fig. 3. JRD contouring of the liver in a series of CT sections of the abdomen. Circles define the initial region samples.

For multiple independent random variables $X_{1}, \ldots, X_{D}$, the $R$-region JRD is

$$
\begin{aligned}
\mathbf{c}_{1}^{*}, \mathbf{c}_{2}^{*}, \ldots, \mathbf{c}_{R-1}^{*}=\arg \max _{\mathbf{c}_{1}, \mathbf{c}_{2}, \ldots, \mathbf{c}_{R-1}} & \\
& {\left[\sum_{d=1}^{D} H_{R \alpha}\left(\sum_{i=0}^{R-1} \pi_{i} p\left(x_{d} \mid Z_{i}\right)\right)-\sum_{i=0}^{R-1} \pi_{i} \sum_{d=1}^{D} H_{R \alpha}\left(p\left(x_{d} \mid Z_{i}\right)\right)\right] }
\end{aligned}
$$

The JRD is optimized by the Nelder-Mead simplex method [18].

\subsection{Random Figure Experiments}

We first used the JRD (equation $(13), R=2$ ) to estimate the contours in a series of random-shape figures created with Fourier elliptic curves 711 with randomized parameters. Figure 2 shows the process: A is a figure template generated with randomized Fourier elliptic coefficients; B shows A smoothed and with added Gaussian noise to a SNR of 4; C shows the initial contour estimate (circle); and D shows the contour computed by the JRD using four Fourier harmonics. The minimum mean square error between corresponding points on the defined and the JRD contours for large sets of images at SNR values from 0.8 to 3.8 were measured and revealed that the smallest average error occured for a Rényi $\alpha \approx 1$. Thus, the minimum error contours could be obtained equivalently by the JSD.

\subsection{Segmentation of Abdominal CT Images}

JRD contours of the liver, and the liver and right kidney are shown in Figures 3 and 4 , respectively, with the circles indicating the initial contour estimates. The multivariate objective function in eq. (13) was used in both cases, using the local pixel mean, variance, and range. The contours expand as a normal consequnce of JRD maximization, and neighboring contours tend not to overlap. 


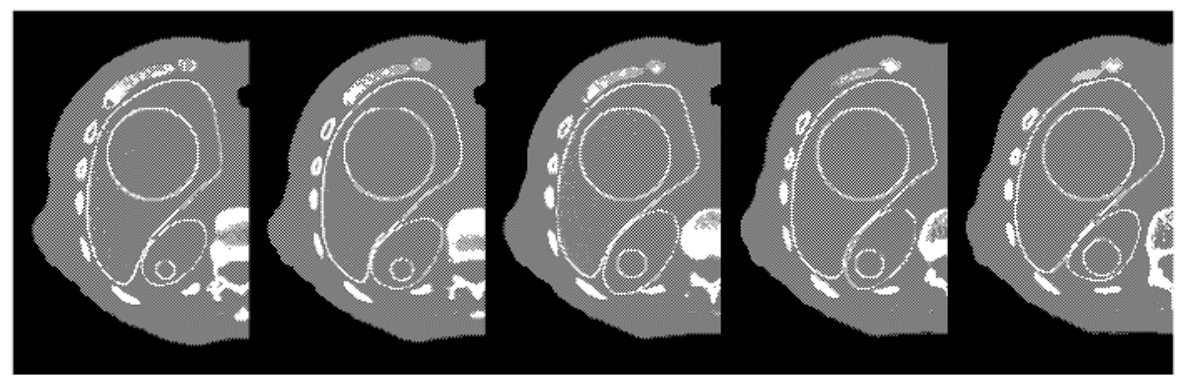

Fig. 4. Simultaneous contouring of the liver and right kidney in a series of CT sections of the abdomen. Circles define the initial region samples.

\section{Conclusions}

The JRD [10] (and its predecessor JSD [14]) have properties making them exemplary objective functions for region segmentation. 1) No model is required for the probablity distributions; 2) two or more regions may be contoured together by optimizing a single function; 3)the JRD is invariant to interchange of region labels; 4)the JRD has no requirement for absolute continuity-all the non-zero probabilities for all regions can be used; 5)the JRD operates only on the marginal distributions of $X_{1}, \ldots, X_{D}$-there is no need to form or process the joint pdf; and 6 )the $\alpha$ parameter may adjust the sensitivity of the discrimination.

Ongoing work will: 1) add JRD-based edge detection and prior shape constraints to equation $(13), 2$ ) compare the discriminatory power of the JRD with ML/MAP methods, and 3) understand the bounded behavior of the JRD with respect to classification error and the Euclidean distance between distributions, in the manner of Lin's [14] analysis of the JSD.

\section{References}

1. A. Collignon, D. Vandermeulen, et al.: Automated multimodality medical image registration using information theory. CVRMed'95, Lecture Notes in Computer Science 905 195-204, Springer-Verlag (1995).

2. T.M. Cover and J.A. Thomas: Elements of Information Theory Wiley-Interscience, New York (1991).

3. L. Devroye, L. Gyorfi and G. Lugosi: A Probabilistic Theory of Pattern Recognition Springer, New York (1996).

4. R.O. Duda, P.E. Hart, and D.G. Stork: Pattern Classification, 2nd Ed., WileyInterscience, New York (2001).

5. K. Fukunaga: Introduction to Statistical Pattern Recognition. Academic Press, New York (1990).

6. J.A. Garcia, J. Fdez-Valdivia, X. Fdez-Vidal and R. Rodriguez-Sanchez: Information theoretic measure for visual target distinctness. IEEE Transactions on Pattern Analysis and Machine Intelligence 23 (2001) 362-383. 
7. C.R. Giardina and F.P. Kuhl: Accuracy of curve approximation by harmonically related vectors with the elliptical loci. Computer Graphics and Image Processing. 21 (1977) 277-285.

8. E. Gokcay and J.C. Principe: Information theoretic clustering. IEEE Transactions on Pattern Analysis and Machine Intelligence. 24 (2002) 158-171.

9. J.F. Gomez-Lopera, J. Martinez-Aroza, A.M. Robles-Perez and R. Roman-Roldan: An Analysis of Edge Detection by Using the Jensen-Shannon Divergence. Journal of Mathematical Imaging and Vision. 13 (2000) 35-56.

10. Y. He, A. Ben Hamza and H. Krim: An information divergence measure for ISAR image registration. IEEE Workshop on Statistical Signal Processing. Singapore, August 2001, 130-133.

11. F.P. Kuhl and C.R. Giardina: Elliptic Fourier features of a closed contour. Computer Graphics and Image Processing. (1982) 18, 236-258.

12. S. Kullback and R.A. Leibler: On information and sufficiency. Annals of Mathematical Statistics. 22 (1951) 79-86.

13. S. Kullback: Information Theory and Statistics. John Wiley and Sons, New York (1959).

14. J. Lin: Divergence measures based on the Shannon entropy. IEEE Transactions on Information Theory. 37 (1991) 145-151.

15. F. Meas, A. Collignon, D. Vandermeulen, G. Marchal, and P. Seutens: Multimodality image registration by maximization of mutual information. IEEE Transactions on Medical Imaging (1997) 16 187-198.

16. J.A. Nelder and R. Mead: A simplex method for function minimization. The Computer Journal. 7 (1965) 308-313.

17. J.A. O'Sullivan, R.E. Blahut, and D.L. Snyder: Information-theoretic image formation. IEEE Transactions on Information Theory 44 (1998) 2094-2123.

18. W.H. Press, S.A. Teukolsky, W.T. Vettering, and B.P. Flannery: Numerical Recipes in $C++$, 2nd Edition Cambridge University Press, Cambridge, UK (2002)

19. J.C. Principe, D. Xu, and J.W. Fisher, III: Information-theoretic learning. in Unsupervised Adaptive Filtering, S. Haykin, Ed. Wiley, New York (1999) 265-319.

20. A. Rényi: On measures of entropy and information. Proceedings of the Fourth Berkeley Symposium on Mathematical Statistics and Probability. Berkeley, CA, June 20-July 30, 1960, Volume I, 547-561 (1961).

21. C.E. Shannon: A mathematical theory of communication. The Bell System Technical Journal. 27 (1948) 379-423.

22. D.L. Snyder, T.J. Schultz, and J.A O'Sullivan: Deblurring subject to nonnegativity constraints. IEEE Transactions on Signal Processing. 40 (1992) 1143-1150.

23. D.L. Snyder, J.A. O'Sullivan, B.R Whiting, R.J. Murphy, J. Benac, J.A. Cataldo, D.G. Politte, and J.F. Williamson: Deblurring subject to nonnegativity constraints when known functions are present with application to object-constrained computerized tomography. IEEE Transactions on Medical Imaging 20 (2001) 1009-1017.

24. C. Studholme, D.L.G. Hill, and D.J. Hawkes: Automated 3-D registration of MR and CT images of the head. Medical Image Analysis 1 (1996) 163-175.

25. P. Viola, and W.M. Wells, III: Alignment by maximization of mutual information. Proc. of the Vth Int'l. Conf. on Computer Vision Cambridge, MA, June, 16-23 (1995).

26. W.M. Wells, III, P. Viola, H. Atsumi, S. Nakajima and R. Kikinis: Multi-modal volume registration by maximization of mutual information. Medical Image Analysis. 1 (1996) 33-51. 